\title{
Parasitismo da cochonilha-farinhenta Planococcus citri (Hemiptera: Pseudococcidae) por Coccidoxenoides perminutus (Hymenoptera: Encyrtidae) ${ }^{\mathbf{1}}$
}

\author{
Karen Oliveira de Menezes ${ }^{2 *}$, Maria Herlândia de Araújo Fernandes ${ }^{3}$, Huanna Húbia Rodrigues Paz , Adriana \\ Maria de Souza ${ }^{5}$, Jorge Braz Torres ${ }^{6}$, José Eudes de Morais Oliveira
}

10.1590/0034-737X201764050005

\begin{abstract}
RESUMO
Planococcus citri é uma importante praga da videira, no submédio do Vale do São Francisco, e seu parasitismo por Coccidoxenoides perminutus, de ocorrência natural nesta região, foi estudado por meio da determinação dos parâmetros de resposta funcional. O experimento foi conduzido com ninfas de segundo ínstar dessa cochonilha, ofertadas nas densidades de 2, 4, 8, 14, 28, 40 e 50, empregando discos de folhas de videira (cv. Syrah). Fêmeas recém-emergidas e alimentadas do parasitoide foram confinadas com as ninfas por 24h. A taxa de parasitismo resultou em resposta funcional do tipo III, com os parâmetros tempo de manipulação $\left(\mathrm{T}_{\mathrm{h}}\right)$ e taxa de ataque $\left(a^{\prime}\right)$ de $0,9824 \mathrm{~h}$ e $0,00718 \mathrm{~h}^{-1}$, respectivamente. Observou-se correlação de $94,3 \%$ entre o parasitismo observado e o estimado pelo modelo de resposta funcional, com máximo de 27 e 24,44 ninfas parasitadas em $24 \mathrm{~h}$, respectivamente. A emergência de parasitoides de ninfas mumificadas variou entre 64 e 95\%, em função das densidades estudadas. Os resultados mostraram que a resposta funcional de $C$. perminutus em ninfas de $P$. citri é do tipo III e que a taxa de emergência do parasitoide é influenciada pelas diferentes densidades de ninfas de $P$. citri oferecidas. Com base na resposta funcional determinada, C. perminutus apresenta potencial de controle da praga, contribuindo com informações para um futuro programa de controle biológico.
\end{abstract}

Palavras-chave: interação parasitoide-hospedeiro; praga da videira; resposta funcional.

\section{ABSTRACT}

\section{Parasitism of the mealybug Planococcus citri (Hemiptera: Pseudococcidae) by Coccidoxenoides perminutus (Hymenoptera: Encyrtidae)}

Planococcus citri is an important pest of vine in the Submedium São Francisco River Valley and its parasitism by Coccidoxenoides perminutus, which naturally occurs in this region, was studied by determining the parameters of functional response. The experiments were carried out using grape leaf (cv. Syrah) disks with second instar mealybug at densities of 2, 4, 8, 14, 28, 40, and 50 nymphs. Newly emerged and fed females were confined with the nymphs for 24 $\mathrm{h}$. The parasitism rate resulted in functional response of type III with the parameters handling time (Th) and attack rate $\left(a^{\prime}\right)$ of $0.9824 \mathrm{~h}$ and $0.00718 \mathrm{~h}^{-1}$, respectively. A correlation of $94.3 \%$ between parasitism observed and estimated by the functional response model was observed, with a maximum of 27 and 24.44 nymphs parasitized in $24 \mathrm{~h}$, respectively. The results showed that the parasitoid exhibited a density-dependent response between 2 and 28 nymphs under the

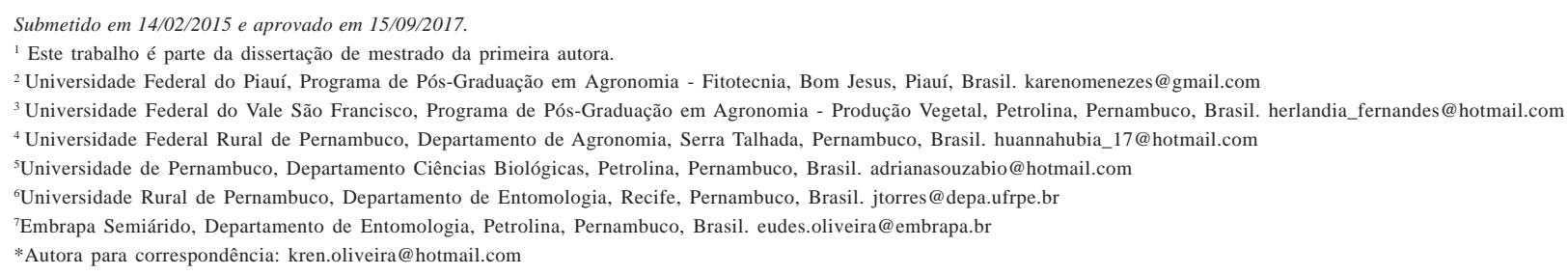


studied conditions. Also, emergence of parasitoids from mummified nymphs were 64 to $95 \%$ and varied as a function of the densities used. The results showed that the functional response of $C$. perminutus to nymphs of $P$. citri is type III and that the rate of emergence of the parasitoid is influenced by the different densities of $P$. citri nymphs offered. Based on the functional response determined, $C$. perminutus presents potential for pest control, contributing with information for a future program of biological control.

Key words: interaction parasitoid-host; pest of vineyard; functional response.

\section{INTRODUÇÃO}

A cochonilha-farinhenta Planococcus citri (Risso, 1813) (Hemiptera: Pseudococcidae) é uma espécie polífaga, com ampla distribuição geográfica, considerada praga para diversas culturas de importância econômica no Brasil, entre as quais algodão, café, citros, hortaliças, manga, plantas ornamentais e videira (Santa-Cecília et al., 2007; Goldasteh et al., 2009; Souza et al., 2012; Ben-Dov et al., 2013).

$\mathrm{Na}$ videira, $P$. citri causa injúrias diretas e indiretas. Ao se alimentarem em raízes, tronco, folhas, ramos e bagas, ocasionam perdas de produção (Daane et al., 2008; Morandi Filho, 2008). Indiretamente, são ainda mais importantes por causa da dispersão de viroses para a viticultura (Cid et al., 2007).

As altas infestações de cochonilhas-farinhentas têm exigido dos produtores aplicações de inseticidas de largo espectro, principalmente, fosforados (Nagrare et al., 2011). Esses produtos são pouco seletivos, altamente tóxicos e apresentam grande intervalo de carência, podendo deixar resíduos nos frutos (Botton et al., 2007), o que reforça o interesse em reduzir o uso desses inseticidas e justifica o estudo de alternativas para o controle dessa praga, como a conservação e uso de agentes de controle biológico.

O sucesso na redução de uma praga por meio do controle biológico depende da eficácia de seus inimigos naturais. O impacto deles sobre a praga alvo está relacionado com a densidade e sua habilidade em encontrar e atacar seus alvos (Jervis \& Kidd, 1996). Dentre as maneiras de avaliar a capacidade dos inimigos naturais como agentes de controle biológico, tem sido utilizada a determinação da resposta funcional. Essa é uma relação entre o número de presas ou hospedeiros atacados em função da densidade, considerando-se o espaço e o intervalo de tempo fixos (Solomon, 1949; Fernández-Arhex \& Corley, 2004). A resposta funcional caracteriza a habilidade dos inimigos naturais em atacar uma população de praga em função da sua densidade. Seus três principais tipos, baseados na taxa de ataque para predadores e parasitoides, são o aumento linear da taxa de ataque (tipo I), a desaceleração da taxa de ataque (tipo II), ou a relação sigmoide da taxa de ataque (tipo III) (Holling, 1959; Hassell, 1978). Fundamentam-se em dois parâmetros básicos que são o tempo de manipulação da presa ou hospedeiro $\left(T_{h}\right)$, e a taxa de ataque (a'). O primeiro representa o encontro, morte e ingestão e a segunda envolve a eficiência de procura, representando o número de presas ou hospedeiros atacados em uma dada área pelo tempo de exposição. $\mathrm{Na}$ modelagem utilizada para determinar a resposta funcional, o principal interesse é a forma da curva que define o tipo de resposta e, consequentemente, o potencial do inimigo natural em regular a população da praga (Beddington et al., 1976; Hassell, 1978); seus parâmetros $\mathrm{T}_{\mathrm{h}}$ e a' (Fan \& Petitt, 1994) definem essa resposta e podem ser empregados para estimar a ação do agente de controle biológico em variadas densidades da praga.

Recentemente, no submédio do Vale do São Francisco foi registrada a presença do parasitoide Coccidoxenoides perminutus Girault, 1915 (Hymenoptera: Encyrtidae), atuando sobre a cochonilha P. citri (Fernandes et al., 2016). Este é um endoparasitoide uniparental que parasita todos os ínstares da cochonilha-farinhenta (Bartlett, 1977). Esse parasitoide foi introduzido em áreas da África do Sul, Bermudas, Chile, Havaí e Peru para o controle biológico dessa praga (Noyes \& Hayat, 1994). Considerando-se o histórico de uso de C. perminutus em outros países, este inseto poderá ser alternativa para o controle dessa praga também no Brasil, especialmente no submédio do Vale do São Francisco onde sua ocorrência é natural. O conhecimento sobre a resposta funcional de C. perminutus, parasitando $P$. citri, poderá fornecer dados acerca de seus parâmetros comportamentais e permitir estimativas que poderão indicar o potencial de sucesso desse parasitoide em função da densidade da praga. Assim, objetivou-se com este trabalho determinar a taxa de parasitismo de $P$. citri por C. perminutus em função da oferta de diferentes densidades dessa cochonilha.

\section{MATERIAL E MÉTODOS}

O experimento foi conduzido no Laboratório de Entomologia da Embrapa Semiárido, em câmaras climatizadas tipo B.O.D. a $25 \pm 1{ }^{\circ} \mathrm{C}$, fotoperíodo $12 \mathrm{~h}$ de L:E e UR de aproximadamente 70\%. O delineamento utilizado foi o inteiramente casualizado, com sete tratamentos (densidades das ninfas) e 20 repetições de cada densidade. 
A taxa de parasitismo de $C$. perminutus sobre $P$. citri foi determinada empregando-se ninfas de segundo ínstar, pois testes preliminares mostraram que esse parasitoide tem preferência por esta idade do hospedeiro, apesar de parasitar todos os ínstares da praga. Para a obtenção das ninfas, 50 fêmeas adultas de $P$. citri, provenientes da criação estoque de laboratório foram transferidas, na densidade de duas fêmeas, para discos foliares de videira (cultivar Syrah), dispostos sobre ágar solidificado no interior de placas de Petri de 5,5 cm de diâmetro $\mathrm{x} 1 \mathrm{~cm}$ de altura. As fêmeas foram retiradas após 48 horas, deixando-se apenas as posturas. Essas posturas foram observadas diariamente com microscópio estereoscópico (40x) para acompanhamento do desenvolvimento das ninfas. A confirmação do segundo ínstar foi concretizada com base nas exúvias. Os discos foliares foram transferidos para novas placas com ágar, sempre que necessário.

Discos de folha de videira ( $3 \mathrm{~cm}$ de diâmetro) foram dispostos sobre ágar solidificado, vertido em placas de Petri (5 $\mathrm{cm}$ de diâmetro). Em seguida, ninfas de segundo ínstar foram transferidas nas densidades de 2, 4, 8, 14, 28, 40 e 50 ninfas por disco. As densidades foram aferidas no dia seguinte à infestação e uma fêmea do parasitoide $C$. perminutus $(\leq 24$ horas de emergência), previamente alimentada com "honeydew", foi mantida por período de 24 horas. O parasitoide foi coletado da criação de $P$. citri e não possuía qualquer experiência de oviposição. As placas foram vedadas com filme plástico de PVC e levadas para câmara climatizada, logo após a liberação do parasitoide.

O parasitoide foi retirado após 24 horas e o parasitismo foi quantificado de acordo com as "múmias" formadas (ninfas parasitadas) após sete e 15 dias. Essas "múmias" foram individualizadas em cápsulas gelatinosas, acondicionadas em câmara climatizada e monitoradas até a emergência do parasitoide. As médias de percentagem de emergência foram comparadas pelo teste de Tukey, a 5\% de probabilidade, pelo software estatístico R versão 2.14.2. Para o cálculo dos parâmetros de resposta funcional, foi utilizado o programa estatístico SAS (SAS Institute 2002), adaptando-se o protocolo escrito por Juliano (1993).

\section{RESULTADOS E DISCUSSÃO}

O parasitismo de ninfas de segundo ínstar de $P$. citri pelo parasitoide $C$. perminutus nas densidades de duas a 50 ninfas resultou em curva sigmoide e caracterizou resposta funcional do Tipo III (Tabela1, Figura 1). Esse tipo de resposta apresentou relação dependente da densidade, sendo que, até o ponto (assíntota) densidade de 28 ninfas/parasitoide, a taxa de parasitismo é crescente (coeficiente linear positivo) de acordo com a densidade do hospedeiro. Assim, mesmo que somente até a densidade de 28 ninfas por parasitoide, a relação parasitoide/hospedeiro apresentou característica de regulação populacional (Van Lenteren \& Bakker, 1976) e demonstrou o potencial desse parasitoide para controle de $P$. citri. Inimigos naturais exibindo resposta funcional do tipo III são capazes de regular a população alvo dentro de certa variação na sua densidade populacional (Beddington et al., 1976; Hassell, 1978).

A resposta funcional de parasitoides atacando pseudococcídeos em laboratório tem variado entre resposta funcional do tipo II (Tripathi \& Singh, 1991) ou tipo III (Pandey et al., 1984). O parasitoide Anagyrus kamali (Hymenoptera: Encyrtidae) parasitando Maconellicoccus hirsutus (Hemiptera: Pseudococcidae), sob condições de tempo fixo e tempo variável, apresentou resposta funcional do tipo II e III em tempo fixo e, apenas do tipo III em tempo variável (Sagarra, 2000). Anagyrus loecki (Hymenoptera: Encyrtidae), parasitando Phenacoccus madeirensis (Hemiptera: Pseudococcidae), exibiu resposta funcional do tipo II (Chong \& Oetting, 2006).

A resposta funcional do tipo III, determinada para $C$. perminutus, parasitando ninfas de $P$. citri é estimada com base em quatro parâmetros: $T_{h}=0,9824 h$ e a' $=0,00718 h^{-1} \mathrm{e}$ as constantes $c$ e $d$. Estes dois últimos parâmetros (estimativa e IC a 95\%) foram $c=0,0371(-0,48-0,55)$ e $d=$ $0,0303(-0,10-0,16)$, que incluíram valores negativos em seu intervalo de confiança a $95 \%$; portanto, não tiveram influência significativa, sendo subtraídos do modelo final (Juliano, 1993). Os parâmetros tempo de manipulação $\left(T_{h}\right)$ e taxa de ataque (a') ou eficiência de procura, definem a resposta funcional e podem variar de acordo com as condições do estudo. A taxa de ataque representa a quantidade de área explorada para encontrar o hospedeiro por unidade de tempo e, portanto, é dependente da distribuição do hospedeiro no ambiente e da dimensão do ambiente. Por outro lado, o tempo de manipulação é característica intrínseca do parasitoide em manipular e matar o hospedeiro por meio da oviposição ou, apenas, pelo ato da alimentação; isso depende das características do hospedeiro, como sua defesa contra parasitismo e modo de reprodução, como parasitoides sinovigênicos. Parasitoides Hymenoptera podem matar o hospedeiro, introduzindo o ovipositor e alimentando-se da hemolinfa extravasada sem realizar oviposição (Jervis \& Kidd, 1986). Assim, a taxa de ataque e o tempo de manipulação são parâmetros que, apesar de sofrerem influência de outros fatores, definem quando a curva de resposta atinge a assíntota e, consequentemente, sua máxima eficiência durante o tempo de experimentação (t) (Mackauer, 1983).

A curva representando a resposta funcional de $C$. perminutus, parasitando ninfas de segundo ínstar de $P$. citri, com chance de re-encontrar uma ninfa já parasitada pode ser expressa pela equação sigmoide, após redução 
dos parâmetros $c$ e $d$ como sendo: $\mathrm{Na}=\exp \left(-a\right.$ ' $\mathrm{TP}_{\mathrm{t}} \mathrm{N}_{0} /$ $\left.1+a \mathrm{~T}_{\mathrm{h}} \mathrm{N}_{0}^{2}\right)$ ] (Hassell, 1978), empregando-se o número inicial de ninfas $\left(\mathrm{N}_{0}\right)$ ofertadas e os parâmetros $\mathrm{T}_{\mathrm{h}}$ e $a$ ' estimados. Considerando-se o uso de uma fêmea do parasitoide por repetição $(\mathrm{Pt}=1)$ e o tempo de experimen- tação $(\mathrm{T}=24 \mathrm{~h})$, a equação de resposta funcional pode ser escrita como: $\left.\mathrm{Nâ}=\exp \left(-0,17232 \mathrm{~N}_{0} / 1+0,00705 \mathrm{~N}_{0}{ }^{2}\right)\right]$. Tendo em vista que a relação $\mathrm{T} / \mathrm{T}_{\mathrm{h}}$ define o número de hospedeiros que podem ser parasitados no tempo de exposição do estudo, o modelo estima que uma média de 24,44 ninfas

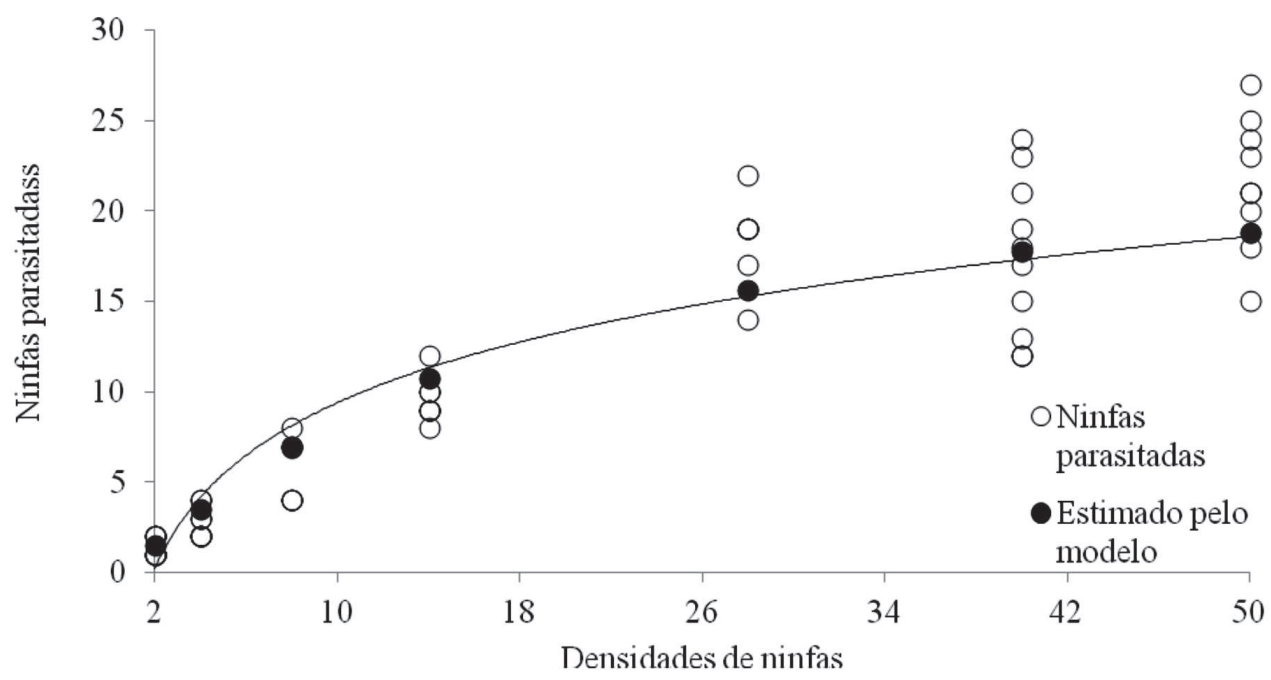

Figura 1: Representação da curva de resposta funcional de Coccidoxenoides perminutus (Hymenoptera: Encyrtidae), parasitando ninfas de segundo ínstar de Planococcus citri (Hemiptera: Pseudococcidae), com período de parasitismo de 24h.

Tabela 1: Resultados da regressão logística da análise da proporção de ninfas de Planococcus citri (Hemiptera: Pseudococcidae) parasitadas por Coccidoxenoides perminutus (Hymenoptera: Encyrtidae), em função da densidade inicial de ninfas, obtidos por meio do Proc CATMOD do SAS (Juliano, 1993)

\begin{tabular}{lcccc}
\hline Coeficientes & Estimativa $( \pm$ EP) & G. L. & $\boldsymbol{\chi}^{\mathbf{2}}$ & Valor de P \\
\hline Intercepto & $0,2621 \pm 0,037$ & 1 & 0,49 & 0,4852 \\
Linear & $0,1218 \pm 0,057$ & 1 & 4,46 & 0,0346 \\
Quadrático & $-0,00601 \pm 0,0027$ & 1 & 7,01 & 0,0081 \\
Cúbico & $0,000067 \pm 0,000026$ & 1 & 6,87 & 0,0088 \\
\hline
\end{tabular}

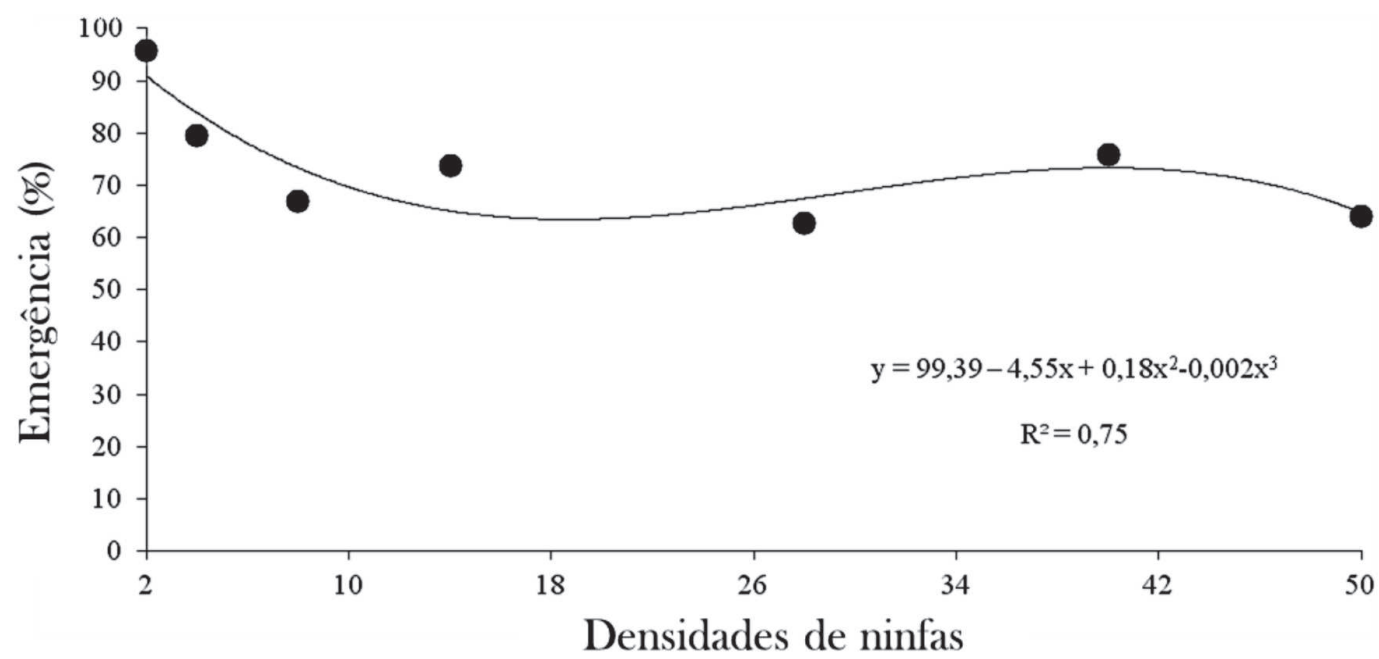

Figura 2: Percentagem de emergência de Coccidoxenoides perminutus (Hymenoptera: Encyrtidae), em função de diferentes densidades de ninfas de Planococcus citri (Hemiptera: Pseudococcidae), em folhas de videira (cv. Syrah) $\left(25 \pm 1{ }^{\circ}\right.$ C, U.R. de $70 \pm 10 \%$ e fotoperíodo de 12L:12E). 
de segundo ínstar $(24 \mathrm{~h} / 0,9824 \mathrm{~h}=24,44$ ninfas*dia $)$ é parasitada durante $24 \mathrm{~h}$ de exposição (12:12h L:E; 70\% UR); enquanto o número máximo de ninfas parasitadas observadas foi de 27 ninfas em uma repetição, na maior densidade testada (50 ninfas).

As médias ( $\pm \mathrm{DP})$ de parasitismo na menor (2 ninfas) e maior (50 ninfas) densidades estudadas foram 1,3 $\pm 0,14 \mathrm{e}$ $21,5 \pm 1,22$, respectivamente. A partir do modelo calculado, estima-se parasitismo médio de 1,54 a 18,81 ninfas. Portanto, o parasitismo estimado correlacionou-se em 94,3\% com o parasitismo observado. Após o parasitismo, as ninfas continuaram se desenvolvendo até mumificarem, o que ocorreu entre sete e 15 dias após o parasitismo. As ninfas parasitadas mostraram hábito de procurar local protegido, movimentando-se para baixo do disco de folha sobre o ágar. A taxa de emergência do parasitoide apresentou diferença $(F=3,04 ; p=0,01)$, em função do aumento das densidades de ninfas, com médias variando de 95,8 a 64,2\% de emergência, para a menor ( 2 ninfas) e maior densidade (50 ninfas), respectivamente (Figura 2).

\section{CONCLUSÕES}

O parasitoide C. perminutus parasita e desenvolve-se em P. citri. A resposta funcional de C. perminutus em ninfas de P. citri é do tipo III, nas condições do estudo. A taxa de emergência do parasitoide é influenciada pelas diferentes densidades de ninfas de P. citri oferecidas. Logo, concluise que o parasitoide C. perminutus apresenta potencial para controle biológico da praga P. citri.

\section{AGRADECIMENTOS}

Os autores agradecem à Coordenação de Aperfeiçoamento de Pessoal de Nível Superior (CAPES), pela bolsa concedida à primeira autora. À Fundação de Amparo à Ciência e Tecnologia de Pernambuco (FACEPE), pelo suporte financeiro, e à Embrapa Semiárido, pela viabilidade na condução da pesquisa. Ao Dr. Valmir Antonio Costa (IB, São Paulo) pela identificação do parasitoide.

\section{REFERÊNCIAS}

Bartlett BR (1977) Citrus mealybug. In: Clausen CP (Ed.) Introduced parasites and predators of arthropod pests and weeds: a World Review. Washington, Agricultural Research Service. p.150-155.

Beddington JR, Hassell ME \& Lawton JH (1976) The components of arthropod predation. II. The predator rate of increase. Journal of Animal Ecology, 45:165-186.

Ben-Dov Y, Miller DR \& Gibson GAP (2013) ScaletNet. Disponível em: 〈http://www.sel.barc.usda.gov/scalenet.html>. Acessado em: 04 de julho de 2014.

Morandi Filho WJ, Botton M, Grutzmacher AD, Fajardo TVM \& Prado E (2007) Vetor encoberto - cochonilhas algodonosas em videira. Cultivar Hortaliças e Frutas, 7:28-29.
Chong JH \& Oetting RD (2006) Functional response and progeny production of the Madeira mealybug parasitoid Anagyrus sp. nov. nr. sinope: the effects of host and parasitoid densities. Biological Control, 39:320-328.

Cid M, Pereira S, Cabaleiro C, Faoro F \& Segura N (2007) Presence of Grapevine leafroll-associated virus 3 in primary salivary glands of the mealybug vector Planococcus citri suggests a circulative transmission mechanism. European Journal of Plant Pathology, 118:23-30.

Daane KM, Cooper ML, Triapitsyn SV, Walton VM, Yokota GY, Haviland DR, Bentley WJ, Godfrey KE \& Wunderlich LR (2008) Vineyard managers and researchers seek sustainable solutions for mealybugs, a changing pest complex. California Agriculture, 62:167-176

Fan Y \& Petitt FL (1994) Parameter estimation of functional response. Environmental Entomology, 23:785-794.

Fernandes MHA, Oliveira JEM, Costa VA \& Menezes KO (2016) Coccidoxenoides perminutus parasitizing Planococcus citri on vine in Brazil. Ciência Rural, 46:1130-1133

Fernández-Arhex V \& Corley JC (2004) The functional response of parasitoids and its implications for biological control. Biocontrol Science and Technology, 13:403-413.

Goldasteh S, Talebi AA, Fathipour Y, Ostovan H, Zamani A \& Shoushtari RV (2009) Effect of temperature on life history and population growth parameters of Planococcus citri (Homoptera, Pseudococcidae) on coleus [Solenostemon scutellarioides (L.) CODD.]. Archives of Biological Sciences, 61:329-336.

Hassell MP (1978) The dynamic of arthropod predator-prey systems. Princeton, Princeton University Press. 237p.

Holling CS (1959) Some characteristics of simple types of predation and parasitism. Canadian Entomologist, 9:385-398.

Jervis MA \& Kidd NAC (1986) Host-feeding strategies in hymenopteran parasitoids. Biological Reviews, 61:395-434.

Jervis M \& Kidd N (1996) Insect Natural Enemies: practical approaches to their study and evaluation. London, Chapman \& Hall. 491p.

Juliano SA (1993) Nonlinear curve fitting: predation and fuctional response curves. In: Scheiner SM \& Gurevitch J (Eds.) Design and Analysis of Ecological Experiments. New York, Chapman and Hall. p.159-182.

Mackauer M (1983) Quantitative assessment of Aphidius smithi (Hymenoptera: Aphiidae): fecundity, intrinsic rate of increase, and functional response. Canadian Entomologist, 115:399-415.

Morandi Filho WJ, Grützmacher AD, Botton M \& Bertin A (2008) Biologia e tabela de vida de fertilidade de Planococcus citri em diferentes estruturas vegetativas de cultivares de videira. Pesquisa Agropecuária Brasileira, 43:941-947.

Nagrare VS, Kranthi S, Kumar R, Dhara Jothi B, Amutha M, Deshmukh AJ, Bisane KD \& Kranthi KR (2011)) Compedium of cotton mealybugs. Disponível em: <http://www.cicr.org.in/ pdf/compendium_of_cotton_mealybugs.pdf $>$. Acessado em: 20 de julho de 2014.

Noyes JS \& Hayat M (1994) Oriental mealybug parasitoids of the Anagyrini (Hymenoptera: Encyrtidae). Disponível em: <http:/ /journals.cambridge.org/action/displayAbstract?fromPage $=$ online $\&$ aid $=2492660>$. Acessado em: 20 de julho 2014 .

Pandey KP, Singh R \& Tripathi CPM (1984) Functional response of Diaeretiella rapae (M'Intosh) (Hym., Aphidiidae), a parasitoid of the mustard aphid Lipaphis erysimi Kalt. (Hom., Aphididae). Journal of Applied Entomology, 98:321-327.

Solomon ME (1949) The natural control of animal populations. The Journal of Animal Ecology, 18:01-35. 
Sagarra LA, Vincent C, Peters NF \& Stewart RK (2000) Effect of host density, temperature, and photoperiod on the fitness of Anagyrus kamali, a parasitoid of the hibiscus mealybug, Maconellicoccus hirsutus. Entomologia Experimentalis et Applicata, 96:141-147.

Santa-Cecília LVC, Souza B, Souza JC, Prado E, Moino Junior A, Fornazier MJ \& Carvalho GA (2007) Cochonilhas-farinhentas em cafeeiros: bioecologia, danos e métodos de controle. Belo Horizonte, Epamig. 48p. (Boletim técnico, 79).
Souza ALV, Souza B, Santa-Cecília LVC \& Prado E (2012) Especificidade alimentar: em busca de um caráter taxonômico para a diferenciação de duas espécies crípticas de cochonilhas do gênero Planococcus (Hemíptera: Pseudococcidae). Revista Brasileira de Fruticultura, 34:744-749.

Tripathi RN \& Singh R (1991) Aspects of life-table studies and functional response of Lysiphelebia minzai. Entomologia Experimentalis et Applicata, 59:279-287.

Van Lenteren JC \& Bakker K (1976) Functional response in invertebrates. Netherlands Journal of Zoology, 26:567-572. 